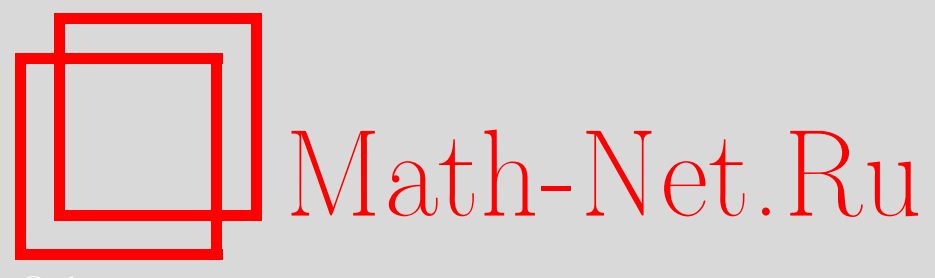

В. И. Солодовников, Бент-функции из конечной абелевой группы в конечную абелеву группу, Дискрет. матем., 2002, том 14, выпуск 1, 99-113

DOI: https://doi.org/10.4213/dm234

Использование Общероссийского математического портала Math-Net.Ru подразумевает, что вы прочитали и согласны с пользовательским соглашением http: //www . mathnet.ru/rus/agreement

Параметры загрузки:

IP : 3.89 .185 .249

26 апреля 2023 г., 14:38:45

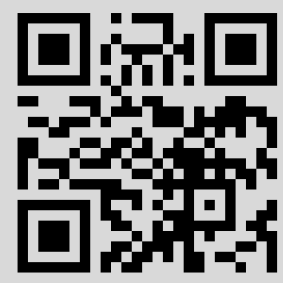




\title{
Бент-функции из конечной абелевой группы в конечную абелеву группу
}

(c) 2002 г. В. И. Солодовников

\begin{abstract}
Вводятся понятия абсолютно негомоморфной функции, минимальной (минимально близкой к гомоморфизмам) функции и бент-функции. Доказывается совпадение класса бент-функций с классом абсолютно негомоморфных функций, однозначность определения функции ее расстояниями до гомоморфизмов со сдвигами, абсолютная минимальность бент-функций для примарного случая.
\end{abstract}

\section{1. Введение}

В работе приняты следующие обозначения и терминология. Будем обозначать $X, Y$ произвольные аддитивные конечные абелевы группы (за исключением теоремы 1 ), на которые по ходу рассуждений накладываются дополнительные ограничения, а $Y^{X}$ - множество всех отображений $f: X \rightarrow Y$ из множества $X$ в множество $Y$ (термины отображение и функция считаем синонимами). Через $\psi \varphi$ обозначается композиция отображений, при которой $\varphi$ действует первым. Пусть $\operatorname{Hom}(G, H)-$ множество всех гомоморфизмов группы $G$ в группу $H$, Aut $(G)-$ группа всех автоморфизмов группы $G, \mathbf{Z}, \mathbf{C}$ - соответственно кольцо целых и поле комплексньх чисел, а

$$
\left(\varphi_{1}, \varphi_{2}\right)=\sum_{x} \varphi_{1}(x) \bar{\varphi}_{2}(x)
$$

- скалярное произведение комплекснозначных функций $\varphi_{1}$ и. $\varphi_{2}$.

Пусть P обозначает равномерное вероятностное распределение на $X$, то есть

$$
\mathbf{P}(A)=|A||X|^{-1}
$$

для любого $A \subseteq X$.

Расстояние $\rho$ между функциями и близость $\delta$ функций $f_{1}, f_{2} \in Y^{X}$ определяются равенствами

$$
\begin{aligned}
& \rho\left(f_{1}, f_{2}\right)=\mathbf{P}\left(f_{1} \neq f_{2}\right), \\
& \delta\left(f_{1}, f_{2}\right)=\left(|Y|^{-1} \sum_{y \in Y}\left(\mathbf{P}\left(f_{1}-f_{2}=y\right)-|Y|^{-1}\right)^{2}\right)^{1 / 2}
\end{aligned}
$$


а расстояние между классами и близость классов функций $K_{1}, K_{2} \subseteq Y^{X}$ определим равенствами

$$
\begin{aligned}
& \rho\left(K_{1}, K_{2}\right)=\min _{f_{1} \in K_{1}, f_{2} \in K_{2}} \rho\left(f_{1}, f_{2}\right), \\
& \delta\left(K_{1}, K_{2}\right)=\max _{f_{1} \in K_{1}, f_{2} \in K_{2}} \delta\left(f_{1}, f_{2}\right) .
\end{aligned}
$$

Функцию называют сбалансированной, если мощности всех прообразов одинаковы.

Введенная формулой (1) близость $\delta$ обладает следующими очевидными свойствами:

$$
\begin{gathered}
\delta\left(f_{1}, f_{2}\right)=\delta\left(f_{2}, f_{1}\right), \\
\left\{\delta\left(f_{1}, f_{2}\right)=0\right\} \Longleftrightarrow\left\{f_{1}-f_{2}-\text { сбалансированная функция }\right\} \\
\delta\left(y_{1}+g_{2} f_{1}+f, y_{2}+g_{2} f_{2} g_{1}+f\right)=\delta\left(f_{1}, f_{2}\right)
\end{gathered}
$$

для любых $f_{1}, f_{2}, f \in Y^{X}$, подстановки $g_{1}$ множества $X, g_{2} \in \operatorname{Aut}(Y), y_{1}, y_{2} \in Y$. Столь же очевидно следующее свойство сбалансированности. Если $f: X \rightarrow Y-$ сбалансированная функция и $h: Y \rightarrow Y^{\prime}$, то $h f-$ сбалансированная функция тогда и только тогда, когда $h-$ сбалансированная функция.

Для любого $a \in X$ подстановку $x \rightarrow a+x$ множества $X$ будем для простоты обозначать также через $a$ (из контекста всегда будет ясно, о чем идет речь, о подстановке $a$ или об элементе $a$ ). Все такие подстановки называют сдвигами группы $X$, и они образуют изоморфную группе $X$ группу подстановок, называемую группой сдвигов (или группой Кэли) группы $X$ (в теории представлений групп такое представление группы $X$ называют регулярным).

Введем теперь понятие абсолютной негомоморфности, к которому можно прийти следующими рассуждениями. Гомоморфизмы $h \in \operatorname{Hom}(X, Y)$ по определению таковы, что $h-h a$ есть постоянная для всех $a \in X \backslash\{0\}$. Тогда функцию такую, что $f-f a$ является сбалансированной функцией для всех $a \in X \backslash\{0\}$, естественно назвать абсолютно негомоморфной, и мы приходим к следующему определению.

Определение 1. Функцию $f: X \rightarrow Y$ назовем абсолютно негомоморфной, если для любого $a \in X \backslash\{0\}$ функция $f-f a$ является сбалансированной, то есть (в силу (3))

$$
\delta\left(f_{1}, f_{2} a\right)=0
$$

Множество всех абсолютно негомоморфных функций из $Y^{X}$ обозначим $B(X, Y)$.

$\mathrm{K}$ понятию абсолютной негомоморфности можно подойти и с другой стороны. $\mathrm{A}$ именно, пусть $G$ - некоторая группа подстановок множества $X, f G=\{f g \mid g \in G\}$ - класс функций, порожденных функцией $f \in Y^{X}$ и группой $G$. Тогда величины $\delta\left(f g_{1}, f g_{2}\right), g_{1} g_{2} \in G$, характеризуют степень изменения функции $f$ подстановками из $G$ : чем ближе эти величины к нулю, тем сильнее подстановки из $G$ изменяют функцию $f$. Поскольку в силу (4)

$$
\delta\left(f g_{1}, f g_{2}\right)=\delta\left(f, f g_{2} g_{1}^{-1}\right),
$$

достаточно рассматривать только величину $\delta(f, f g), g \in G$. В частности, когда $G$ - группа сдвигов группы $X$, получаем, что абсолютно негомоморфные функции это функции, которые максимально изменяются сдвигами. 
Более полной характеристикой схожести функций, чем близость $\delta$, является соответствующее распределение. В связи с этим найдем среднее значение величины $\mathbf{P}\left(f_{1}-f_{2} g=y\right)-|Y|^{-1}$ при случайном равновероятном выборе $g$ из $G$ (в этой теореме $X$ - произвольное множество, $Y$ - произвольная группа с аддитивной записью операции).

Теорема 1. Пусть $f_{1}, f_{2} \in Y^{X}, Y$ - аддитивная группа, $G$ - группа подстановок множества $X, \operatorname{Orb}(G)$ - множество всех орбит группы $G$. Тогда для любого у $\in Y$

$$
\begin{aligned}
& |G|^{-1} \sum_{g \in G}\left(\mathbf{P}\left(f_{1}-f_{2} g=y\right)-|Y|^{-1}\right) \\
& \quad=|X|^{-1} \sum_{T \in \operatorname{Orb}(G)}|T| \sum_{y^{\prime} \in Y}\left(\mathbf{P}\left(f_{1}=y+y^{\prime} \mid T\right)-|Y|^{-1}\right)\left(\mathbf{P}\left(f_{2}=y^{\prime} \mid T\right)-|Y|^{-1}\right) .
\end{aligned}
$$

Доказательство. Пусть $\Delta$ - символ Кронекера. Справедлива цепочка равенств

$$
\begin{aligned}
\sum_{g \in G} \mathbf{P}\left(f_{1}-f_{2} g=y\right) & =\sum_{g \in G} \sum_{y^{\prime} \in Y} \mathbf{P}\left(f_{1}=y+y^{\prime}, f_{2} g=y^{\prime}\right) \\
& =\sum_{g \in G} \sum_{y^{\prime} \in Y}|X|^{-1} \sum_{x \in X} \Delta\left(f_{1}(x), y+y^{\prime}\right) \Delta\left(f_{2} g(x), y^{\prime}\right) \\
& =|X|^{-1} \sum_{T \in \operatorname{Orb}(G)} \sum_{x \in T} \sum_{y^{\prime} \in Y} \sum_{g \in G} \Delta\left(f_{1}(x), y+y^{\prime}\right) \Delta\left(f_{2} g(x), y^{\prime}\right) \\
& =|X|^{-1} \sum_{T \in \operatorname{Orb}(G)} \sum_{y^{\prime} \in Y} \sum_{x \in T} \Delta\left(f_{1}(x), y+y^{\prime}\right) \sum_{g \in G} \Delta\left(f_{2} g(x), y^{\prime}\right) \\
& =|X|^{-1} \sum_{T \in \operatorname{Orb}(G)} \sum_{y^{\prime} \in Y} \sum_{x \in T} \Delta\left(f_{1}(x), y+y^{\prime}\right)|G||T|-1 \sum_{x \in T} \Delta\left(f_{2}(x), y^{\prime}\right) \\
& =|G||X|^{-1} \sum_{T \in \operatorname{Orb}(G)} \sum_{y^{\prime} \in Y} \sum_{x \in T} \Delta\left(f_{1}(x), y+y^{\prime}\right) \mathbf{P}\left(f_{2}=y^{\prime} / T\right) \\
& =|G||X|^{-1} \sum_{T \in \operatorname{Orb}(G)} \sum_{y^{\prime} \in Y}|T| \mathbf{P}\left(f_{1}=y+y^{\prime} \mid T\right) \mathbf{P}\left(f_{2}=y^{\prime} / T\right),
\end{aligned}
$$

то есть

$$
|G|^{-1} \sum_{g \in G} \mathbf{P}\left(f_{1}-f_{2} g=y\right)=|X|^{-1} \sum_{T \in \operatorname{Orb}(G)}|T| \sum_{y^{\prime} \in Y} \mathbf{P}\left(f_{1}=y+y^{\prime} \mid T\right) \mathbf{P}\left(f_{2}=y^{\prime} \mid T\right) .
$$

Далее доказательство очевидно. Теорема доказана.

Следствие 1. Если при условиях теоремы 2 әруппа $G$ транзитивна, то

$$
|G|^{-1} \sum_{g \in G}\left(\mathbf{P}(f=f g)-|Y|^{-1}\right)=|Y| \delta^{2}(f, 0) .
$$

Приведем некоторые свойства абсолютно негомоморфных функций.

Предложение 1. Если $a \in X, b \in Y, g_{1} \in \operatorname{Aut}(X), g_{2} \in \operatorname{Aut}(Y), h \in \operatorname{Hom}(X, Y) u$ $f \in B(X, Y)$, mo $b+h+g_{2} f\left(a+g_{1}\right) \in B(X, Y)$.

Утверждение следует из (4) и определения 1. 
Предложение 2. Если $f \in B(X, Y), h: Y \rightarrow Y^{\prime}$ - эпиморфизм (то есть сюорвективный гомоморфизм групn), mo $h f \in B\left(X, Y^{\prime}\right)$.

Утверждение следует из определения 1 и сбалансированности $h$.

Предложение 3. Eсли $f \in B(X, Y), h \in \operatorname{Hom}(X, Y)$, mo

$$
\delta^{2}(f, h)=\frac{|Y|-1}{|X||Y|^{2}} .
$$

Утверждение следует из предложения 1 и следствия 1.

Кстати, отсюда следует, что абсолютно негомоморфные функции не являются сбалансированными при $|Y|>1$.

Предложение 4. Пусть $X=X_{1} \times X_{2}$, где $X_{1}, X_{2}$ - аддитивнъе әруппъ,

$$
f_{i}: X_{i} \rightarrow Y, \quad i=1,2, \quad f=f_{1}+f_{2} .
$$

Тогда если одна из функций $f_{1}, f_{2}$ является сбалансированной, то $f$ - сбалансированнал функция $u$

$$
f \in B(X, Y) \Leftrightarrow f_{i} \in B\left(X_{i}, Y\right), \quad i=1,2 .
$$

Доказательство очевидно.

Свойство абсолютной негомоморфности имеет следующую матричную интерпретацию.

Определение 2. Матрицу $\left(y_{a, x}\right)_{a, x \in X}$ размера $|X| \times|X|$ с элементами из множества $Y$, строки и столбцы которой занумерованы элементами группы $X$, назовем $X$-матрицей над $Y$, если $y_{a, x}=y_{a^{\prime}, x^{\prime}}$ для любых $a, x, a^{\prime}, x^{\prime} \in X$ таких, что $a+x=$ $a^{\prime}+x^{\prime}$. Множество всех $X$-матриц над $Y$ обозначим через $M(X, Y)$.

Обозначим $K(X)=(a+x \mid a, x \in X)$ таблицу Кэли группы $X$. Очевидно, что отображение $Y^{X} \rightarrow M(X, Y)$, где

$$
f \rightarrow f(K(X))
$$

является биекцией.

Определение 3. Назовем $X$-матрицу над $Y$ бент-матрицей, если в разности любых двух ее строк с различными номерами все элементы из $Y$ встречаются одинаковое число раз. Множество всех бент-матриц обозначим через $B M(X, Y)$.

Очевидно следующее утверждение.

Предложение 5. Соотночение (6) задает взаимно однозначное соответствие между множествами $B(X, Y)$ и $B M(X, Y)$.

В дальнейшем нам потребуется определение минимальной функции, которое уместно дать уже сейчас.

Определение 4. Функцию $f: X \rightarrow Y$ назовем минимальной, если она минимально близка ко всем гомоморфизмам, то есть

$$
\delta(f, \operatorname{Hom}(X, Y))=\max _{f^{\prime} \in Y^{X}} \delta\left(f^{\prime}, \operatorname{Hom}(X, Y)\right) .
$$


Заметим, что из предложения 3 следует, что абсолютно негомоморфные функции одинаково близки к любому гомоморфизму, а предложение 2 означает, что свойство абсолютной негомоморфности инвариантно относительно любых эпиморфизмов группы $Y$. Последнее наводит нас на следующее определение.

Определение 5. Функцию $f: X \rightarrow Y$ назовем абсолютно минимальной, если для любого эпиморфизма $h: Y \rightarrow Y^{\prime}$ функция $h f: X \rightarrow Y^{\prime}$ минимальна.

Заметим, что минимальные функции существуют всегда, в отличие от абсолютно минимальных или абсолютно негомоморфных функций. В дальнейшем (теорема 8) мы покажем, что для примарного случая класс $B(X, Y)$ содержится в классе всех абсолютно минимальных функций, а для двоичного случая эти классы совпадают.

Далее нам потребуются следующие хорошо известные сведения об абелевых группах.

Всякая конечная аддитивная абелева группа $X$ представляется в виде

$$
X \cong \prod \mathbf{Z} /\left(m_{i}\right)
$$

Поэтому всюду далее будем считать, что

$$
X=\prod_{i=1}^{k} \mathbf{Z} /\left(m_{i}\right), \quad Y=\prod_{j=1}^{t} \mathbf{Z} /\left(n_{j}\right)
$$

где $k, t, m_{1}, \ldots, m_{k}, n_{1}, \ldots, n_{t}$ - произвольные натуральные числа.

Для каждого $\alpha=\left(\alpha_{1}+\left(m_{1}\right), \ldots, \alpha_{k}+\left(m_{k}\right)\right) \in X$ определим комплекснозначную функцию $\chi_{\alpha}: X \rightarrow \mathbf{C}$ равенством

$$
\chi_{\alpha(x)}=\prod_{i=1}^{k} \exp \left\{\left(2 \pi i / m_{i}\right) \alpha_{i} x_{i}\right\}=\exp \left\{2 \pi i \sum_{i=1}^{k} \frac{1}{m_{i}} \alpha_{i} x_{i}\right\}
$$

для всех $x=\left(x_{1}+\left(m_{1}\right), \ldots, x_{k}+\left(m_{k}\right)\right) \in X$. Эти функции обладают следующими свойствами: для любых $\alpha, \alpha^{\prime} \in X$

$\chi_{\alpha}$ - гомоморфизм (относительно умножения в $\mathbf{C}$ );

$\chi_{\alpha} \neq \chi_{\alpha^{\prime}}$ при $\alpha \neq \alpha^{\prime}$;

$\chi_{\alpha}\left(\alpha^{\prime}\right)=\chi_{\alpha^{\prime}}(\alpha)$

$\chi_{0} \equiv 1$.

Для $i=1, \ldots, k$ положим

$$
e_{i}=\left(\left(m_{1}\right), \ldots,\left(m_{i-1}\right), 1+\left(m_{i}\right),\left(m_{i+1}\right), \ldots,\left(m_{k}\right)\right) .
$$

Очевидно, что для любых $\alpha, x, x^{\prime} \in X$

$$
\begin{gathered}
\chi_{\alpha}(x)=\prod_{i=1}^{k}\left(\chi_{e_{i}}(x)\right)^{\alpha_{i}} \\
\left\{\chi_{e_{i}}(x)=\chi_{e_{i}}\left(x^{\prime}\right), i=1, \ldots, k\right\} \Longleftrightarrow\left\{x=x^{\prime}\right\} .
\end{gathered}
$$


Следовательно, отображение $X \rightarrow \mathbf{C}^{k}$, где

$$
x \rightarrow\left(\chi_{e_{1}}(x), \ldots, \chi_{e_{k}}(x)\right)
$$

есть инъективный гомоморфизм. Отсюда следует, что с помощью соответствия (9) всегда можно занумеровать элементы произвольной абелевой группы комплекснозначными векторами, причем такая нумерация будет гомоморфной.

Из теории представлений групп следует, что $\left\{\chi_{\alpha} \mid \alpha \in X\right\}-$ множество всех неприводимых характеров абелевой группы $X$, причем относительно умножения оно является группой, изоморфной группе $X$. Следовательно, выполняются известные соотношения ортогональности

$$
|X|^{-1}\left(\chi_{\alpha}, \chi_{\alpha^{\prime}}\right)=\Delta\left(\alpha, \alpha^{\prime}\right)
$$

для любых $\alpha, \alpha^{\prime} \in X$, что равносильно условию

$$
|X|^{-1} \sum_{\alpha \in X} \chi_{\alpha}(x)=\Delta(x, 0)
$$

для любого $x \in X$.

Свойство (10) означает, что множество $\left\{\chi_{\alpha} \mid \alpha \in X\right\}$ является ортонормированным относительно скалярного произведения $|X|^{-1}(\cdot, \cdot)$ базисом векторного пространства $\mathbf{C}^{X}$ и, следовательно, любая функция $\varphi: X \rightarrow \mathbf{C}$ однозначно представляется в виде

$$
\varphi=\sum_{\alpha \in X} C_{\alpha}^{\varphi} \chi_{\alpha}
$$

где $C_{\boldsymbol{\alpha}}^{\varphi} \in \mathbf{C}$ и

$$
C_{\alpha}^{\varphi}=|X|^{-1}\left(\varphi, \chi_{\alpha}\right)
$$

для всех $\alpha \in X$. Равенство (12) называют разложением Фурье функции $\varphi$, а $C_{\alpha}^{\varphi}$, $\alpha \in X,-$ коэффициентами Фурье функции $\varphi$. Они однозначно определяют функцию $\varphi$ и обладают следующими свойствами:

$$
\begin{aligned}
\left|C_{\alpha}^{\varphi}\right| & \leqslant \max _{x \in X}|\varphi(x)|, \\
C_{0}^{\varphi} & =\mathbf{M} \varphi \\
\sum_{\alpha \in X}\left|C_{\alpha}^{\varphi}\right|^{2} & =\mathbf{M}|\varphi|^{2}
\end{aligned}
$$

где М означает среднее значение. Равенство (16) называют равенством Парсеваля.

Разложение Фурье имеет следующую матричную интерпретацию. Матрицей характеров абелевой группы $X$ называют матрицу $H=\left(\chi_{\alpha}(x)\right)_{\alpha, x \in X}$. Она обладает следующими свойствами:

$\chi_{\alpha}(x) \in \sqrt[m]{1}$, где $m$ - наименьшее общее кратное чисел $m_{1}, \ldots, m_{k}$,

$H$ - симметричная матрица,

$H, \bar{H}=|X| E$, где $E-$ единичная матрица, 
$\chi_{0}(x)=\chi_{\alpha}(0)=1$ для всех $\alpha, x \in X$,

$\chi_{\alpha}(x) \chi_{\alpha^{\prime}}(x)=\chi_{\alpha+\alpha^{\prime}}(x)$ для всех $\alpha, \alpha^{\prime}, x \in X$,

$H=H_{1} \otimes \ldots \otimes H_{k}$, где $H_{i}$ - матрица характеров группы $\mathbf{Z} /\left(m_{i}\right), i=1, \ldots, k$,

преобразование $\varphi \rightarrow C^{\varphi}$, где $C^{\varphi}: \alpha \rightarrow C_{\alpha}^{\varphi}$, множества $\mathbf{C}^{X}$ является автоморфизмом векторного пространства $\mathbf{C}^{X}$, матрица которого относительно стандартного базиса есть $|X|^{-1} H$ (это преобразование называют преобразованием Фурье).

Определение 6. Матрицей Адамара порядка $n$ степени $m$ называют $n \times n$ матрицу $A=\left(a_{i j}\right)$ над множеством $\sqrt[m]{1} \in \mathbf{C}$, обладающую свойством

$$
A \bar{A}^{T}=n E .
$$

Если $a_{1, i}=a_{i, 1}=1$ для всех $i=1, \ldots, n$, то $A$ называют нормализованной матрицей Адамара.

Таким образом, матрица характеров конечной абелевой группы есть симметричная матрица Адамара, множество строк которой замкнуто относительно умножения. Наоборот, всякая симметричная матрица Адамара с замкнутым относительно умножения множеством строк есть матрица характеров группы строк этой матрицы.

Для гомоморфизма $h \in \operatorname{Hom}(X, Y)$ определим $k \times t$ матрицу $A_{h}=\left(a_{i, j}+\left(n_{j}\right)\right)$ гомоморфизма $h$ равенствами

$$
h\left(e_{i}\right)=\left(a_{i, 1}+\left(n_{1}\right), \ldots, a_{i, t}+\left(n_{t}\right)\right), \quad i=1, \ldots, k .
$$

Она обладает следующими свойствами:

$$
n_{j} /\left[m_{i}, n_{j}\right] \mid a_{i, j}
$$

для любых $i=1, \ldots, k, j=1, \ldots, t$ (здесь и далее $\left[r_{1}, \ldots, r_{s}\right]$ - наибольший общий делитель чисел $r_{1}, \ldots, r_{s}$ и $a \mid b$ означает, что $a$ делит $\left.b\right)$,

$$
h\left(\left(x_{1}+\left(m_{1}\right), \ldots, x_{k}+\left(m_{k}\right)\right)\right)=\left(x_{1}, \ldots, x_{k}\right) A_{h}
$$

для любых $x_{1}, \ldots, x_{k} \in \mathbf{Z}$ и, следовательно, матрица $A_{h}$ однозначно определяет гомоморфизм $h$. Наоборот, если $A=\left(a_{i, j}+\left(n_{j}\right)\right)-$ произвольная $k \times t$ матрица, обладающая свойством (17), то соответствие $h_{A}: X \rightarrow Y$, определяемое равенством

$$
h_{A}\left(\left(x_{1}+\left(m_{1}\right), \ldots, x_{k}+\left(m_{k}\right)\right)\right)=\left(x_{1}, \ldots, x_{k}\right) A,
$$

является гомоморфизмом групп. Таким образом, между $\operatorname{Hom}(X, Y)$ и всеми матрицами со свойством (17) существует взаимно однозначное соответствие и, следовательно,

$$
|\operatorname{Hom}(X, Y)|=\prod_{i=1, \ldots, k, j=1, \ldots, t}\left[m_{i}, n_{j}\right] .
$$

Пусть теперь $\left\{\eta_{\beta} \mid \beta \in Y\right\}-$ множество всех неприводимых характеров группы $Y$, определяемое в соответствии с равенством (8) для $Y$. Тогда для любых $\beta \in Y$, $h \in \operatorname{Hom}(X, Y)$ композиция $\eta_{\beta} h$ - гомоморфизм и, следовательно,

$$
\eta_{\beta} h=\chi_{\alpha}
$$


для некоторого $\alpha \in X$. Пусть $\beta=\left(\beta_{1}+\left(n_{1}\right), \ldots, \beta_{t}+\left(n_{t}\right)\right)$ и $A_{h}=\left(a_{i, j}+\left(n_{j}\right)\right)$ - матрица размера $k \times t$. Тогда непосредственно из определений получаем, что равенство (19) выполняется для $\alpha=\left(\alpha_{1}+\left(m_{1}\right), \ldots, \alpha_{k}+\left(m_{k}\right)\right)$, где

$$
\alpha_{i}=\sum_{j=1}^{t} \frac{m_{i} a_{i, j}}{n_{j}} \beta_{j}, \quad i=1, \ldots, k .
$$

В дальнейшем нам потребуется следующая лемма.

Лемма 1. Следующие утверждения равносильны:

(1) $\left\{\eta_{\beta} h \mid \beta \in Y, h \in \operatorname{Hom}(X, Y)\right\}=\left\{\chi_{\alpha} \mid \alpha \in X\right\}$,

(2) $\left\{\eta_{e} h \mid h \in \operatorname{Hom}(X, Y)\right\}=\left\{\chi_{\alpha} \mid \alpha \in X\right\}$, где $e=\left(1+\left(n_{1}\right), \ldots, 1+\left(n_{t}\right)\right)$,

(3) $\left[m_{i} /\left[m_{i}, n_{1}\right], \ldots, m_{i} /\left[m_{i}, n_{t}\right]\right]=1$ для любого $i=1, \ldots, k$,

(4) наименьиее общее кратное чисел $m_{1}, \ldots, m_{k}$ делит наименъиее общее хратное чисел $n_{1}, \ldots, n_{t}$.

Доказательство. Докажем, что (1) $\Rightarrow(3)$. Пусть

$$
\left[m_{i} /\left[m_{i}, n_{1}\right], \ldots, m_{i} /\left[m_{i}, n_{t}\right]\right]=d_{i}>1 \text {. }
$$

Тогда в силу (20) и (17)

$$
\alpha_{i}+\left(m_{i}\right)=\sum_{j=1}^{t}\left(m_{i} /\left[m_{i}, n_{j}\right]\right)\left(a_{i, j} /\left(n_{i} /\left[m_{i}, n_{j}\right]\right) \beta_{j}+\left(m_{i}\right) \neq 1+\left(m_{i}\right)\right.
$$

для любых $\beta \in Y$ и $h \in \operatorname{Hom}(X, Y)$, что противоречит утверждению (1) леммы.

Докажем теперь, что (3) $\Rightarrow(2)$. Из (3) следует, что для любого $i=1, \ldots, k$ найдутся числа $a_{i, 1}^{\prime}, \ldots, a_{i, t}^{\prime} \in \mathbf{Z}$ такие, что

$$
1=\sum_{j=1}^{t} \frac{m_{i}}{\left[m_{i}, n_{j}\right]} a_{i, j}^{\prime}
$$

Тогда для любых $\alpha_{1}, \ldots, \alpha_{k} \in \mathbf{Z}$

$$
\alpha_{i}=\sum_{j=1}^{t} \frac{m_{i} a_{i, j}}{n_{j}}, \quad a_{i, j}=\left(\alpha_{i} \alpha_{i, j}^{\prime}\right) \frac{n_{j}}{\left[m_{i}, n_{j}\right]},
$$

и в силу (17) и (20) условие (2) выполнено.

Наконец, то, что (2) $\Rightarrow(1)$ и (3) $\Leftrightarrow(4)$, очевидно. Лемма доказана.

Пусть всюду далее $\left\{\chi_{\alpha} \mid \alpha \in X\right\}$ и $\left\{\eta_{\beta} \mid \beta \in Y\right\}-$ множества всех неприводимых характеров абелевых групп $X$ и $Y$, определяемые в соответствии с формулой (8). Кроме того, пусть $\left\{\eta_{\beta}^{[j]} \mid \beta \in \mathbf{Z} /\left(n_{j}\right)\right\}$ - множество всех неприводимых характеров группы $\mathbf{Z} /\left(n_{j}\right)$. Очевидно, что

$$
\eta_{\beta}=\prod_{j=1}^{t} \eta_{\beta_{j}}^{[j]}
$$


для любого $\beta=\left(\beta_{1}, \ldots, \beta_{t}\right) \in Y$.

Для любой функции $f: X \rightarrow Y$ через $f^{(1)}, \ldots, f^{(t)}$ будем обозначать координатные функции отображения $f$, то есть $f^{(j)}: X \rightarrow \mathbf{Z} /\left(n_{j}\right)$ и $f(x)=\left(f^{(1)}(x), \ldots, f^{(t)}(x)\right)$ для всех $x \in X$.

Определение 7. Характерами отображения $f: X \rightarrow Y$ будем называть отображения $\eta_{\beta} f: X \rightarrow \mathbf{C}$ для всех $\beta \in Y$. При

$$
\beta=e_{j}=\left(\left(n_{1}\right), \ldots,\left(n_{j-1}\right), 1+\left(n_{j}\right),\left(n_{j+1}\right), \ldots,\left(n_{t}\right)\right), \quad j=1, \ldots, t
$$

назовем их базисными.

В силу (9) базисные характеры однозначно определяют функцию $f$, причем

$$
\eta_{\beta} f=\prod_{j=1}^{t}\left(\eta_{e_{j}} f\right)^{\beta_{j}}
$$

для любого $\beta=\left(\beta_{1}+\left(n_{1}\right), \ldots, \beta_{t}+\left(n_{t}\right)\right) \in Y$, а также

$$
\eta_{e_{j}} f=\eta_{1+\left(n_{j}\right)}^{[j]} f^{(j)}
$$

для любого $j=1, \ldots, t$, так что базисные характеры являются базисными характерами координатных функций.

Определение 8. Производящей функцией отображения $f: X \rightarrow Y$ называется (см. [1]) функция $\varphi_{f}: Y \rightarrow \mathrm{C}$, определяемая равенством

$$
\varphi_{f}(\beta)=M \eta_{\beta} f=|X|^{-1} \sum_{x \in X} \eta_{\beta}(f(x)) .
$$

Очевидно, что

$$
\varphi_{f}=\sum_{y \in Y} \mathbf{P}(f=y) \eta_{y}
$$

и в силу (13)

$$
\mathbf{P}(f=y)=|Y|^{-1}\left(\varphi_{f}, \eta_{y}\right)
$$

для любого $y \in Y$. Следовательно, по производящей функции $\varphi_{f}$ однозначно определяется распределение случайной величины $f$ и, в частности,

$$
\{f-\text { сбалансированная функция }\} \Longleftrightarrow\left\{\varphi_{f}(\beta)=0 \text { для любого } \beta \neq 0\right\} \text {. }
$$

В силу (12) и (13) разложение Фурье характера отображения $f$ имеет вид

$$
\eta_{\beta} f=\sum_{\alpha \in X} C_{\alpha}^{\eta_{\beta} f} \chi_{\alpha}
$$

где

$$
C_{\alpha}^{\eta_{\beta} f}=|X|^{-1}\left(\eta_{\beta} f, \chi_{\alpha}\right)
$$


Отсюда следует, что коэффициенты Фурье базисных характеров функции $f$

$$
C_{\alpha}^{\eta_{e_{j}} f}, \quad j=1, \ldots, t, \quad \alpha \in X
$$

однозначно определяют функцию $f$ (количество этих чисел равно $t|X|)$. Из (14)-(16) получаем, что

$$
\begin{aligned}
\left|C_{\alpha}^{\eta_{\beta} f}\right| & \leqslant 1, \\
C_{0}^{\eta_{\beta} f} & =\varphi_{f}(\beta), \\
\sum_{\alpha \in X}\left|C_{\alpha}^{\eta_{\beta} f}\right|^{2} & =1 .
\end{aligned}
$$

(последнее равенство есть равенство Парсеваля).

Кроме того, очевидны равенства

$$
\begin{aligned}
\varphi_{f_{1}-f_{2}}(\beta) & =\sum_{\alpha \in X} C_{\alpha}^{\eta_{\beta} f_{1}} C_{\alpha}^{\eta_{\beta} f_{2}}, \\
\delta^{2}\left(f_{1}, f_{2}\right) & =|Y|^{-2} \sum_{0 \neq \beta \in Y}\left|\varphi_{f_{1}-f_{2}}(\beta)\right|^{2}, \\
C_{\alpha}^{\eta_{\beta}(b+f a)} & =\eta_{\beta}(b) \chi_{\alpha}(a) C_{\alpha}^{\eta_{\beta} f}
\end{aligned}
$$

для любых $a \in X$ и $b \in Y$, а также

$$
\varphi_{f_{a_{1}}-f_{a_{2}}}(\beta)=\sum_{\alpha \in X}\left|C_{\alpha}^{\eta_{\beta} f}\right|^{2} \chi_{\alpha}\left(a_{1}-a_{2}\right)
$$

для любых $a_{1}, a_{2} \in X$.

Из (34) вытекает следующее утверждение.

Теорема 2. Для любых $f_{1}: X \rightarrow Y$ и $f_{2}: X \rightarrow Y$ следующие утверждения равносильны:

$\mathbf{P}\left(f_{1}-f_{1} a=y\right)=\mathbf{P}\left(f_{2}-f_{2} a=y\right) \partial л я$ любы $a \in X, y \in Y$,

$\left|C_{\alpha}^{\eta_{\beta} f_{1}}\right|=\left|C_{\alpha}^{\eta_{\beta} f_{2}}\right|$ для люобых $\alpha \in X, \beta \in Y$.

Из (30) вытекает следующее утверждение.

Предложение 6. Для любых $f: X \rightarrow Y u \beta \in Y$

$$
\max _{\alpha \in X}\left|C_{\alpha}^{\eta_{\beta} f}\right| \geqslant|X|^{-1 / 2}
$$

причем следующие утверждения равносилъны:

$\max _{\alpha \in X}\left|C_{\alpha}^{\eta_{\beta} f}\right|=|X|^{-1 / 2}$

$\left|C_{\alpha^{\prime}}^{\eta_{\beta} f}\right|=|X|^{-1 / 2}$ для всех $\alpha \in X$,

$\left|C_{\alpha}^{\eta_{\beta} f}\right|=\left|C_{\alpha^{\prime}}^{\eta_{\beta} f}\right|$ для любъх $\alpha, \alpha^{\prime} \in X$. 
Это предложение приводит нас к следующему определению.

Определение 9. Функцию $f: X \rightarrow Y$ назовем бент-функцией, если для любых $\alpha, \alpha^{\prime} \in X, \beta \in Y \backslash\{0\}$

$$
\left|C_{\alpha}^{\eta_{\beta} f}\right|=\left|C_{\alpha^{\prime}}^{\eta_{\beta} f}\right| .
$$

Это определение обобщает определения работ [4] (для булевых функций), [1] (для $\left.X=G F(q)^{n}, Y=G F(q)\right)$ и может быть сведено к определению работы [2], где бент-функции определяются как комплекснозначные функции на конечной абелевой группе с единичными модулями всех значений и условием равномодульности всех коэффициентов Фурье.

Следующая теорема обобщает соответствующие результаты работ [4], [1] и следует из свойства (26) и результатов работы [2].

Теорема 3. Для любой функции $f: X \rightarrow Y$ следующие свойства равносилъны:

(1) $f$ - абсолютно негомоморфная функция,

(2) $f$ - бент-функция,

(3) $\left\{\eta_{\beta} f_{a} \mid a \in X\right\}$ - ортонормированный относителъно $|X|^{-1}(\cdot, \cdot)$ базис пространства $\mathrm{C}^{X}$ для любого $\beta \in Y \backslash\{0\}$.

Доказателъство. В силу определения 1 и свойства (26) условие (1) равносильно условию

$$
\varphi_{f-f a}(\beta)=0
$$

для всех $a \in X \backslash\{0\}, \beta \in Y \backslash\{0\}$, которое в силу определения 8 и теоремы 1 работы [2] равносильно условию

$$
\left|C_{\alpha}^{\eta_{\beta} f}\right|^{2}=|X|^{-1}
$$

для всех $\beta \in Y \backslash\{0\}, \alpha \in X$, и в силу предложения 6 равносильность (1) и (2) доказана. Равносильность (2) и (3) следует из теоремы 2 работы [2]. Теорема доказана.

Заметим, что свойство (3) теоремы 3 приводит к следующей интерпретации бентфункций из $B(X, Y)$ матрицами Адамара. Для каждых $f: X \rightarrow Y$ и $\beta \in Y$ рассмотрим матрицу

$$
A_{\beta, f}=\left(\eta_{\beta} f(a+x)\right)_{a, x \in X}
$$

размера $|X| \times|X|$, строки и столбцы которой занумерованы элементами множества $X$. Эта матрица является $X$-матрицей (определение 2 ), так как

$$
A_{\beta, f}=\eta_{\beta} f(K(X))
$$

где $K(X)$ - таблица Кэли группы $X$. Если $\beta \neq 0$ и $f \in B(X, Y)$, то по теореме 3 матрица $A_{\beta, f}$ - матрица Адамара (определение 2). Обозначим через $A M(X, \sqrt[n]{1})$ множество всех $X$-матриц над $\sqrt[n]{1}$, являющихся матрицами Адамара степени $n$. Тогда из (23), (6) и теоремы 3 следует, что соответствие

$$
B(X, Y) \rightarrow A M\left(X, \sqrt[n_{1}]{1}\right) \times \ldots \times A M\left(X, \sqrt[n_{t}]{1}\right)
$$

где

$$
f \rightarrow\left(\eta_{e_{1}} f(K(X)), \ldots, \eta_{e_{t}} f(K(X))\right),
$$


является инъективным отображением.

Следующая теорема распространяет известный результат работы [3] для булевых функций на случай абелевых групп. Она утверждает, что набор расстояний от функции $f$ до всех функций вида $y+h$, где $y \in Y, h \in \operatorname{Hom}(X, Y)$, однозначно определяет функцию $f$.

Теорема 4. Пусть $m_{i}$ делит $n_{j}$ при любых $i=1, \ldots, k, j=1, \ldots, t$. Тогда для любой $f: X \rightarrow Y$ набор чисел

$$
\{\mathbf{P}(f=y+h) \mid y \in Y, h \in \operatorname{Hom}(X, Y)\}
$$

однозначно определяет функцию $f$.

Доказательство. Покажем, что по указанному набору вероятностей однозначно определяются координатные функции $f^{(1)}, \ldots, f^{(t)}$. Пусть $1 \leqslant j \leqslant t$. Для любого $h^{\prime} \in \operatorname{Hom}\left(X, \mathbf{Z} /\left(n_{j}\right)\right)$ существует $h \in \operatorname{Hom}(X, Y)$ такой, что $h^{(j)}=h^{\prime}$ и, следовательно, мы можем определить числа

$$
\mathbf{P}\left(f^{(j)}=y^{\prime}+h^{\prime}\right)=\sum_{y=\left(y_{1}, \ldots, y_{t}\right) \in Y, y_{j}=y^{\prime}} \mathbf{P}(f=y+h)
$$

для всех $y^{\prime} \in \mathbf{Z} /\left(n_{j}\right), h^{\prime} \in \operatorname{Hom}\left(X, \mathbf{Z} /\left(n_{j}\right)\right)$, то есть производящую функцию $\varphi_{f^{(j)}-h^{\prime}}$. Из того, что $m_{i}$ делит $n_{j}$ при всех $i=1, \ldots, k$, следует, что условие (4) леммы 1 для $X$ и $\mathbf{Z} /\left(n_{j}\right)$ выполнено и, следовательно, для любого $\alpha \in X$ существует гомоморфизм $\left.h^{\prime} \in \operatorname{Hom}^{\prime} X, \mathbf{Z} /\left(n_{j}\right)\right)$ такой, что

$$
\chi_{\alpha}=\eta_{1+\left(n_{j}\right)}^{[j]} h^{\prime}
$$

и в силу (30)

$$
\varphi_{f^{(j)}-h^{\prime}}\left(1+\left(n_{j}\right)\right)=C_{\alpha}^{\eta_{1+\left(n_{j}\right)}^{[j]}} f^{(j)} .
$$

Тем самым, базисный характер функции $f^{(j)}$ определяется однозначно, а следовательно, и $f^{(j)}$ определяется однозначно. Теорема доказана.

Пусть всюду далее $X=(\mathbf{Z} /(m))^{k}, Y=(\mathbf{Z} /(m))^{t}$. Заметим, что этот случай включает рассмотренный в [1] случай $X=G F(q)^{k^{\prime}}, Y=G F(q)^{t^{\prime}}$, так как здесь имеют место изоморфизмы абелевых групп $X \cong(\mathbf{Z} /(p))^{r k^{\prime}}, Y \cong(\mathbf{Z} /(p))^{r t^{\prime}}$. Заметим также, что при $r \geqslant 2$ гомоморфизмов абелевых групп $X \rightarrow Y$ больше, чем линейных отображений $G F(q)$-пространств $X \rightarrow Y$, так как $|\operatorname{Hom}(X, Y)|=p^{r^{2} k^{\prime} t^{\prime}}$, а число линейных отображений равно $p^{r k^{\prime} t^{\prime}}$.

Прежде всего опишем $\operatorname{Hom}(X, Y)$. В соответствии с (18) любой гомоморфизм $h \in \operatorname{Hom}(X, Y)$ однозначно представляется в виде

$$
h\left(\left(x_{1}+(m), \ldots, x_{k}+(m)\right)\right)=\left(x_{1}, \ldots, x_{k}\right) A_{h}
$$

где $k \times t$ матрица $A_{h}=\left(a_{i, j}+(m)\right)$ и числа $a_{i, j}$ могут быть любыми, так что $|\operatorname{Hom}(X, Y)|=m^{k t}$. В силу (20) для любых $h \in \operatorname{Hom}(X, Y), \beta \in Y$

$$
\eta_{\beta} h=\chi_{\beta A_{h}^{T}}
$$


и в силу (34) и (32)

$$
\begin{aligned}
\varphi_{f-h}(\beta) & =C_{\beta A_{h}^{T}}^{\eta_{\beta} f}, \\
\delta^{2}(f, h) & =|Y|^{-2} \sum_{0 \neq \beta \in Y}\left|C_{\beta A_{h}^{T}}^{\eta_{\beta} f}\right|^{2}
\end{aligned}
$$

для любой $f: X \rightarrow Y$.

Следующие две теоремы сводят многомерный случай к одномерному и обобщают теорему 3 из [1].

Теорема 5. Для любой функции $f: X \rightarrow Y$ следующие свойства равносилънъ:

(1) $f$ - сбалансированная функция,

(2) $h f-$ сбалансированная функция для любого эпиморфизма $h: Y \rightarrow Z /(m)$.

Доказателъство. Очевидно, что $(1) \Rightarrow(2)$, так как гомоморфизмы сбалансированы. Докажем, что (2) $\Rightarrow(1)$. Пусть

$$
0 \neq \beta=\left(\beta_{1}+(m), \ldots, \beta_{t}+(m)\right) \in Y, \quad d=\left(\beta_{1}, \ldots, \beta_{t}\right), \quad b=\frac{1}{d} \beta,
$$

и $h_{b}: Y \rightarrow \mathbf{Z} /(m)$ - гомоморфизм с матрицей $b^{T}$. Тогда $h_{b}(Y) \ni 1+(m)$, так что $h_{b}$ сюръективен, причем $\eta_{d+(m)}^{\prime} h_{b}=\eta_{d b}=\eta_{\beta}$. Поскольку $h_{b} f-$ сбалансированная функция и $d \neq 0$, в силу (26) и (29)

$$
0=C_{0}^{\eta_{l+(m)}^{\prime}\left(h_{b} f\right)}=C_{0}^{\eta_{\beta} f}
$$

Поскольку $\beta$ - произвольный ненулевой элемент, снова в силу (26) и (29) $f$ - сбалансированная функция. Теорема доказана.

Из этой теоремы, определения 1 и теоремы 3 сразу получаем следующую теорему. Теорема 6. Для любой функции $f: X \rightarrow Y$ следующие свойства равносильны:

(1) $f$ - бент-функция,

(2) $h f-$ бент-функция для любого эпиморфизма $h: Y \rightarrow \mathbf{Z} /(m)$.

Заметим, что утверждение (2) теоремы 5 (теорема 6) означает, что для любого $c=\left(c_{1}, \ldots, c_{t}\right) \in Y, \quad c \neq 0$,

$$
\sum_{j=1}^{t} c_{j} f^{(j)}
$$

- сбалансированная функция (бент-функция).

Рассмотрим теперь вопрос о том, как связаны понятия бент-функции и минимальной функции (определение 4). Для краткости обозначим

$$
\delta_{0}(X, Y)=\min _{f \in Y^{X}} \delta(f, \operatorname{Hom}(X, Y))
$$

минимальную близость функции к гомоморфизмам. Дальнейшие рассуждения удается провести лишь для случая простого модуля $m$. 
Лемма 2. Если $m$ - простое число, то для любъх $f: X \rightarrow Y, \beta \in Y \backslash\{0\}$

$$
\begin{aligned}
|\operatorname{Hom}(X, Y)|^{-1} \sum_{h \in \operatorname{Hom}(X, Y)}\left|\varphi_{f-h}(\beta)\right|^{2} & =|X|^{-1}, \\
|\operatorname{Hom}(X, Y)|^{-1} \sum_{h \in \operatorname{Hom}(X, Y)} \delta^{2}(f, h) & =\frac{|Y|-1}{|X||Y|^{2}} \\
\delta_{0}(X, Y) & \geqslant\left(\frac{|Y|-1}{|X||Y|^{2}}\right)^{1 / 2} .
\end{aligned}
$$

Доказательство. В силу (36)

$$
m^{-k t} \sum_{h}\left|\varphi_{f-h}(\beta)\right|^{2}=m^{-k t} \sum_{A^{\prime}}\left|C_{\beta A^{\prime}}^{\eta_{\beta} f}\right|^{2}
$$

Отображение $A^{\prime} \rightarrow \beta A^{\prime}$ является гомоморфизмом аддитивной абелевой группы всех $t \times k$ матриц над $\mathbf{Z} /(m)$ в группу $X$. Поскольку $\beta \neq 0$ и $m-$ простое число, этот гомоморфизм сюръективен. Следовательно, в силу (30) это равенство можно продолжить следующим образом:

$$
m^{-k t} \sum_{A^{\prime}}\left|C_{\beta A^{\prime}}^{\eta_{\beta} f}\right|^{2}=m^{-k t} \sum_{\alpha \in X} m^{k t}|X|^{-1}\left|C_{\alpha}^{\eta_{\beta} f}\right|^{2}=|X|^{-1}
$$

и равенство (38) доказано.

В силу (37) и доказанного равенства

$$
\begin{aligned}
m^{-k t} \sum_{h} \delta^{2}(f, h) & =m^{-k t} \sum_{h}|Y|^{-2} \sum_{\beta \neq 0}\left|\varphi_{f-h}(\beta)\right|^{2} \\
& =|Y|^{-2} \sum_{\beta \neq 0} m^{-k t} \sum_{h}\left|\varphi_{f-h}(\beta)\right|^{2}=\frac{|Y|-1}{|X||Y|^{2}}
\end{aligned}
$$

и равенство (39) доказано.

В силу равенства (39), неравенство (40) очевидно. Лемма доказана.

Теорема 7. Если $m$ - простое число, то для любой функции $f: X \rightarrow Y$ следующие свойства равносильны:

(1) $f$ - минимальная функция $\delta_{0}(X, Y)=\left((|Y|-1) /\left(|X||Y|^{2}\right)\right)^{1 / 2}$,

(2) $\delta(f, h)=\delta\left(f, h^{\prime}\right) \partial л я л ю б ъ x ~ h, h^{\prime} \in \operatorname{Hom}(X, Y)$,

(3) $\delta(f, h)=\left((|Y|-1) /\left(|X||Y|^{2}\right)\right)^{1 / 2} \partial л я$ яюбого $h \in \operatorname{Hom}(X, Y)$.

Доказательство. Докажем, что (1) $\Rightarrow(3)$. По определению минимальности

$$
\max _{h \in \operatorname{Hom}(X, Y)} \delta^{2}(f, h)=\frac{|Y|-1}{|X||Y|^{2}}
$$

и свойство (3) выполнено в силу равенства (39) леммы 2.

Соотношение $(3) \Rightarrow(2)$ очевидно. 
Наконец, докажем, что (2) $\Rightarrow(1)$. Из равенства (39) следует, что

$$
\delta(f, \operatorname{Hom}(X, Y))=\left(\frac{|Y|-1}{|X||Y|^{2}}\right)^{1 / 2}
$$

и по определению

$$
\delta_{0}(X, Y) \leqslant\left(\frac{|Y|-1}{|X||Y|^{2}}\right)^{1 / 2} .
$$

Тогда в силу (40), свойство (1) выполняется. Теорема доказана.

Теорема 8. Если $m$ - простое число и $B(X, Y) \neq \varnothing$, то любая бент-функция является абсолютно минимальной $u$

$$
\delta_{0}(X, Y)=\left(\frac{|Y|-1}{|X||Y|^{2}}\right)^{1 / 2}
$$

Eсли $m=2$ и $B(X, Y) \neq \varnothing$, то в $Y^{X}$ класс всех бент-функций совпадает $c$ классом всех абсолютно минимальных функций.

Доказательство. Первое утверждение теоремы является очевидным следствием предложения 3, утверждения (3) из теоремы 7 и предложения 2.

Докажем второе утверждение. Пусть функция $f: X \rightarrow Y$ абсолютно минимальна и $h: Y \rightarrow \mathbf{Z} /(m)$ - произвольный эпиморфизм. Тогда $h f$ минимальна, и в силу предложения 3 , теоремы 7 и (37)

$$
|X|^{-1} 2^{-2}=\delta^{2}\left(h f, h^{\prime}\right)=2^{-2}\left|C_{\alpha}^{\eta_{\beta} h f}\right|^{2}
$$

для любого гомоморфизма $h: X \rightarrow \mathbf{Z} /(m)$, где $\beta=1+(2), \alpha=A_{h^{\prime}}^{T} \in X$. Тогда $h f$ - бент-функция. Поскольку $h$ - произвольный эпиморфизм, по теореме $6 f-$ бент-функция. Теорема доказана.

Наконец, отметим, что в [1] доказано, что при простом $m$, если $2 t$ делит $k$ или $t$ делит $k$ при $m \neq 2$, то $B(X, Y) \neq \varnothing$.

\section{Список литературы}

1. Амбросимов А. С., Свойства бент-функций $q$-значной логики над конечными полями. Дисхретная математиха (1994) 6, №3, 216-226.

2. Логачев О. С., Сальников А. А., Ященко В. В., Бент-функции на конечной абелевой группе. Дискретная математика (1997) 9, №4, 3-20.

3. Golomb S. W., On the classification of Boolean functions. IRE Trans. Circuit Theory (1959) 1, №6, 10-27.

4. Rothaus O. S., On "bent" functions. J. Comb. Theory, Ser. A (1976) 20, №3, 300-305.

Статья поступила 18.02.2000. Переработанный вариант поступил 13.02.2001. 